\title{
Dye monolayers used as hole transporting medium in dye sensitized solar cells
}

Davide Moia,${ }^{1{ }^{*}}$ Tomas Leijtens, ${ }^{2 \ddagger}$ Nakita Noel, ${ }^{2}$ Henry J. Snaith, ${ }^{2}$ Jenny Nelson, ${ }^{1}$ and Piers R. F. Barnes ${ }^{1 *}$

1. Blackett Laboratory, Imperial College London, London SW7 2AZ, UK

2. Clarendon Laboratory, University of Oxford, OX1 3PU, UK

${ }^{\ddagger}$ these authors contributed equally

\section{*davide.moia11@imperial.ac.uk; piers.barnes@imperial.ac.uk}

The transport of holes in molecular monolayers attached to mesoporous metal oxide surfaces has been observed both electrochemically ${ }^{[1],[2]}$ and spectroscopically ${ }^{[3]}$. Its demonstration has shown that dye sensitization is a unique route towards tailoring interfacial electrical properties of oxide mesostructures. The phenomenon has been applied as molecular wiring in batteries, ${ }^{[4]}$ as a means to collect charges to catalytic centers on photoelectrodes for solar fuel production. ${ }^{[5]}$ It has also been suggested as one of the mechanisms occurring in dye sensistized solar cells (DSSCs) especially those utilizing hole transport materials which cannot completely fill the mesoporous $\mathrm{TiO}_{2}$ anode. ${ }^{[6],[7],[8],[9],[10],[11]}$ In our previous studies we discussed the regime of hole transport between dyes anchored to $\mathrm{TiO}_{2}$ films immersed in inert electrolytes in terms of chemical structure of the dye and molecular fluctuations. ${ }^{[12],[13]}$ Here we directly demonstrate that intermolecular hole hopping through dye monolayers is sufficient to make working photovoltaic devices based on the DSSC architecture without the infiltration of a redox electrolyte or hole transport material to regenerate the oxidized dyes. The 'dry cells' showed surprisingly high incident photon conversion efficiencies (IPCEs), reaching $13 \%$. This was possible because the molecules anchored to the surface serve multiple functions within the device: absorbing light and injecting the resulting photoexcited electrons into the metal oxide semiconductor, followed by the collection of the remaining holes through intermolecular charge transport to the counter electrode in the surface monolayer. The addition of an electron blocking layer under the metal contact resulted in open circuit voltages approaching $1 \mathrm{~V}$. The measurements illustrate the potential of molecular conductive coatings using multi-functional molecules, and suggest interesting new possibilities for the construction of organic electronic devices and sensors.

Figure 1 shows the architectures examined: an FTO glass substrate was coated with a compact $\mathrm{TiO}_{2}$ layer, this was covered by a 0.7 to $2 \mu \mathrm{m}$ thick photoactive layer formed from mesoporous film of $\mathrm{TiO}_{2}$ nanocrystals sensitized with the indoline dyes D131 or D149. A metal contact (silver or gold) was evaporated as the counter electrode. In some cases, a semitransparent contact was used ( $40 \mathrm{~nm}$ thick), enabling illumination from both sides of the device. Although we refer to this concept as to a 'dry cell' all measurements were performed in air, so the term 'dry' should not to be taken literally, as water is expected to be present on the surface of the $\mathrm{TiO}_{2}$ in ambient conditions. To further verify the role of hole transport between dyes in photocurrent generation, we also investigated architectures where an additional insulating nanocrystalline $\mathrm{Al}_{2} \mathrm{O}_{3}$ layer $(0.15 \mu \mathrm{m})$ was deposited on top of $0.9 \mu \mathrm{m}$ thick $\mathrm{TiO}_{2}$ film before sensitization. This layer blocks transfer of electrons to the counter electrode and also prevents direct regeneration of oxidized dye molecules on the $\mathrm{TiO}_{2}$ by the metal. More details on the sample preparation and photovoltaic characterization can be found in the supporting information. 


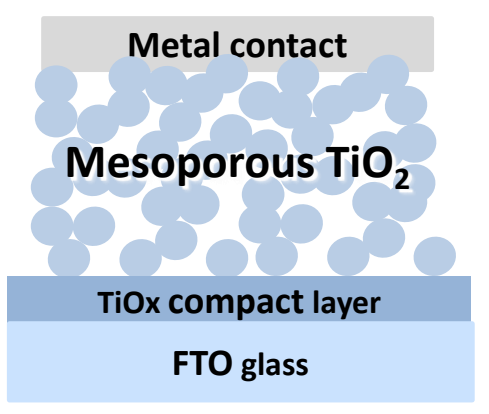

a

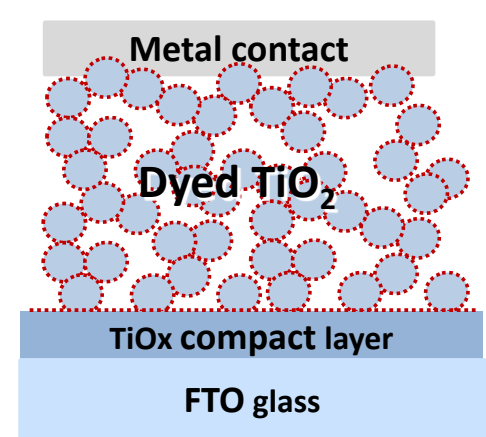

b

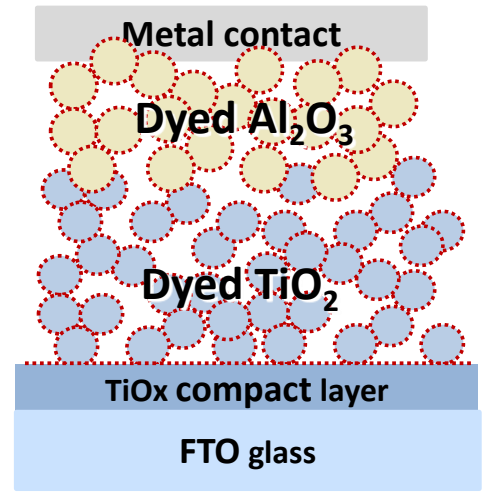

C

Figure 1. Solar cell devices investigated in this study: (a) mesoporous $\mathrm{TiO}_{2}$ 'dry cell' where no dye or other hole transport material (HTM) is included in the device; (b) dye sensitized $\mathrm{TiO}_{2}$ 'dry cell' where the dye monolayer acts as $\mathrm{HTM}$; (c) dye sensitized $\mathrm{TiO}_{2}$ 'dry cell' including a dye sensitized $\mathrm{Al}_{2} \mathrm{O}_{3}$ mesoporous film. 


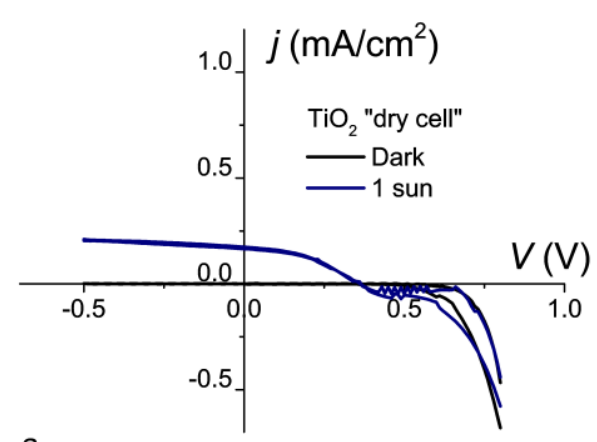

a

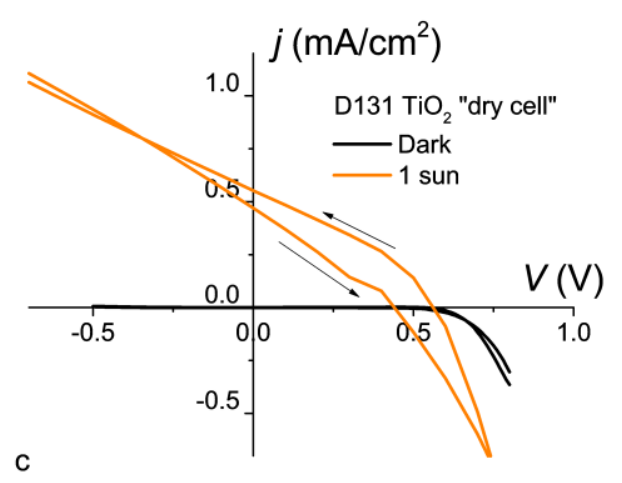

c

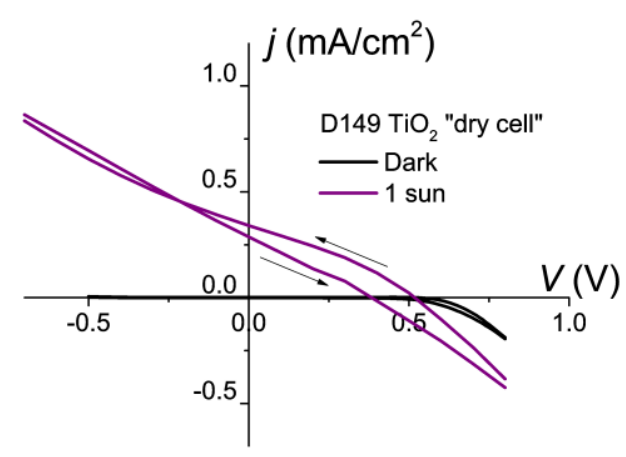

e

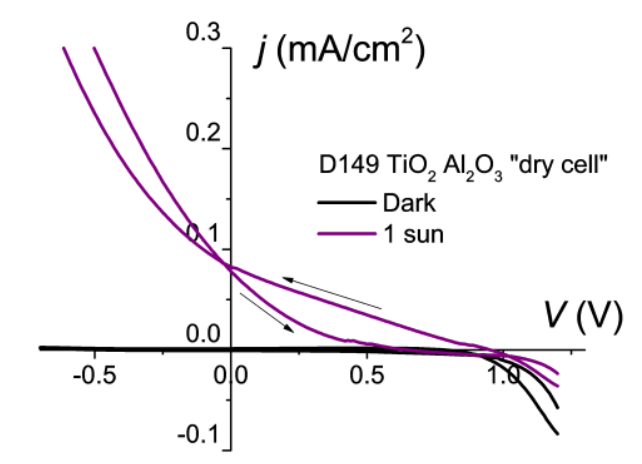

g

Figure 2. Current density-voltage and IPCE measurements performed on $(a, b)$ unsensitized $\mathrm{TiO}_{2}$, (c - d) $\mathrm{D} 131,(\mathrm{e}, \mathrm{f}) \mathrm{D} 149$ sensitized $\mathrm{TiO}_{2}$ "dry cells" and $(\mathrm{g}, \mathrm{h}) \mathrm{D} 149$ sensitized "dry cells" including a dyed mesoporous alumina layer. $j$ - $V$ characterization was carried out under simulated AM1.5 sunlight. The IPCE measurements were performed with a LED bias light at 0.1 sun equivalent intensity and the illumination of the device was directed to the FTO glass side or to the semitransparent silver or 
gold contact side. An estimate of the absorptance spectrum of the absorber $\left(\mathrm{TiO}_{2}\right.$ in $b$, dyes in $d, f$ and $h$ ) is also shown to be compared with the IPCE profiles.

Figure 2 shows results for devices which have a semitransparent metal contact. IPCE measured under front (FTO) and back (metal counter electrode) side illumination are shown. Figures $2 \mathrm{a}$ and $2 \mathrm{~b}$ show the control measurements made on an unsensitized mesoporous $\mathrm{TiO}_{2}$ film. The dark $j-V$ curve indicated rectifying behaviour from the device and, on illumination with a simulated AM1.5 spectrum, a photocurrent and photovoltage were observed. The presence of a space charge region in the $\mathrm{TiO}_{2}$ in proximity of either contact would explain this observation. This would assist the separation of photogenerated holes and electrons formed from optical excitations across the anatase band gap. The shape of the $j-V$ curve close to $V_{o c}$ suggests that a barrier is formed at both the $\mathrm{TiO}_{2} / \mathrm{Ag}$ and the $\mathrm{TiO}_{2} /$ FTO interfaces. The action of UV light on the device is confirmed by the IPCE spectrum which is greatest (about 25\%) at $350 \mathrm{~nm}$ when the device is illuminated from the FTO side and tracks the absorptance profile of anatase above its band gap. The lower value of IPCE for the Ag side illumination observed for this particular device suggests that efficient charge separation in the $\mathrm{TiO}_{2}$ semiconductor occurs mainly close to the interface with FTO.

Figures $2 c-2 f$ show the photovoltaic characterization for the $\mathrm{D} 131$ and $\mathrm{D} 149$ dye sensitized $\mathrm{TiO}_{2}$ devices. Rectifying behaviour was again observed in the dark in most cases. The 'dry cells' showed remarkable photocurrent and a clear contribution to the IPCE corresponding to the dye absorption (also shown) was observed. The value of IPCE below $400 \mathrm{~nm}$ was found to decrease when compared to the unsensitized devices. $\mathrm{TiO}_{2}$ strongly absorbs at short wavelengths. Any photogenerated holes in the $\mathrm{TiO}_{2}$ are likely to be transferred to the dyes in the sensitized devices. For different batches of devices we observe a diminished but non-zero response at these wavelengths for dye sensitized films compared to unsensitized controls. This is consistent with less efficient collection of photogenerated holes through the dye monolayer than through the valence band of $\mathrm{TiO}_{2}$. Working dry cells were also fabricated using the ruthenium dye $\mathrm{Z907}$ which showed lower photocurrents (see supporting information). Previously we have found this dye to show similar values for the hole apparent diffusion coefficient to D131 in acetonitrile based electrolytes. ${ }^{[12]}$ The lower performance of $\mathbf{Z 9 0 7}$ dry solar cells is ascribed to the lower extinction coefficient of the dye (and thus poor light harvesting in the $\mathrm{TiO}_{2}$ thickness available) compared to indolene dyes.

We note that some hysteresis is evident in the $j-V$ curves (which were scanned at about $0.15 \mathrm{~V} \mathrm{~s}^{-1}$ ). This could be due to chemical capacitance effects and charge density dependent electron mobility in the $\mathrm{TiO}_{2}$. Further analysis of the dependence of hysteresis on scan rate is presented in the supporting information. This observation implies that the measurement is far from being a steady state assessment of the device performance in terms of power conversion efficiency. The $j_{s c}$ exceeded $1 \mathrm{~mA} \mathrm{~cm}{ }^{-2}$ for the best performing device (shown in figure 3a), and values of IPCE up to $13 \%$ (see figure $3 \mathrm{~b}$ ) were measured. The devices were sufficiently stable for photo-electrical characterization. For example, figure $3 \mathrm{c}$ shows multiple scans performed on the same device in the dark or under 1 sun illumination. Decrease in $V_{o c}$ and FF is observed, while the short circuit current held to a consistent value over the 5 scans in the light. The photocurrent in these devices showed a strong dependence on electrical bias: it increased almost linearly with reverse bias, in contrast to the same devices in the dark and the unsensitized control device. A large spread in the distribution of photocurrent values was observed. Also, some of the 'dry cells' showed low shunt resistance, possibly due to the presence of pinholes, for devices having either Au or Ag as metal contact. Spontaneous shunting was occasionally observed during current voltage measurements, when scanning the voltage of devices towards forward bias in the dark or under illumination. This is likely to be related to the failure of the rectifying properties of the $\mathrm{TiO} 2 / \mathrm{dye} / \mathrm{Ag}$ interface (see supporting information). 


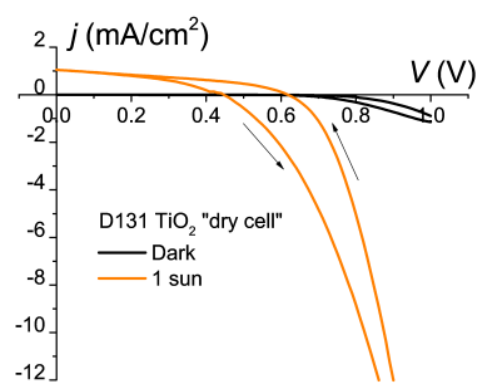

a

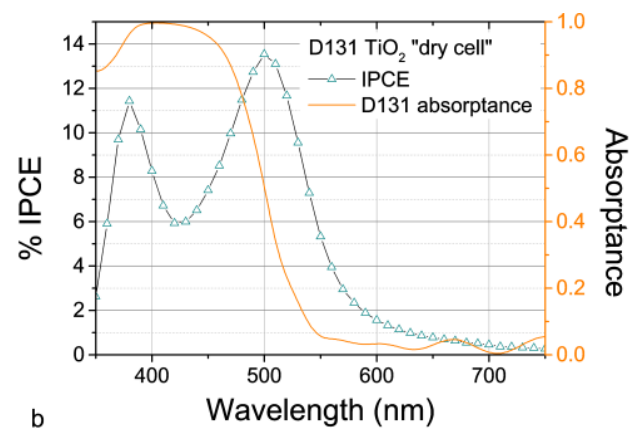

$b$

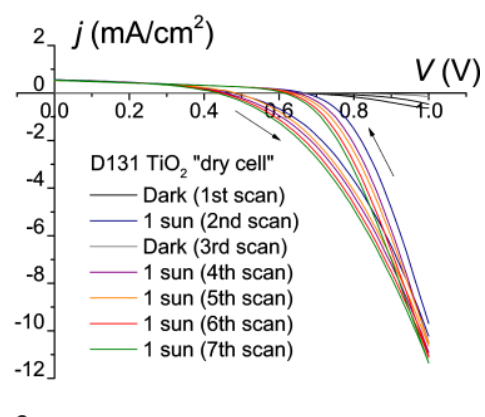

Figure 3. $(\mathrm{a}, \mathrm{b}) j-V$ and IPCE measurements on the best performing 'dry cell'. (c) Multiple $j-V$ measurement on a $\mathrm{D} 131$ sensitized $\mathrm{TiO}_{2}$ 'dry cell'.

The observed photocurrent could be explained in terms of regeneration of dyes in direct contact with the metal electrode, which would not require any hole hopping through the dye layer. DSSCs made of a monolayer of dyes on a flat $\mathrm{TiO}_{2}$ layer have been demonstrated in the past suggesting that this can be an efficient process. ${ }^{[14]}$ In our case the evaporated metal layer would have to be in contact with a sensitized area corresponding to more than $200 \mathrm{~nm}$ of the top layer of particles in order to account for the IPCE spectrum shown in figure $3 \mathrm{c}$ by direct regeneration (model shown in the supporting information). For this conservative estimate we have also assumed specular back reflection of the capping section of the metal contact and $100 \%$ electron injection efficiency, thus it seems very unlikely that direct regeneration by the metal gives a dominant contribution to the photocurrent. This conclusion is supported by the thickness dependence of the photocurrent in the device showing an optimum for values around $1 \mu \mathrm{m}$, and also by the analysis of the IPCE spectra which were best described by assuming poor charge collection from the dyed $\mathrm{TiO}_{2}$ metal interface (see supporting information).

To confirm that direct regeneration by the metal contact is not required, devices with an additional dyed $\mathrm{Al}_{2} \mathrm{O}_{3}$ electron blocking layer on top of the titania mesoporous film were characterized following a similar approach. Devices with this architecture also showed a strong increase in photocurrent under reverse bias. For the case of D149, the photocurrent at short circuit was high enough (figure $2 \mathrm{~g}$ ) to enable the measurement of the device's spectral response which can be seen to correspond to the absorption spectrum of the dye (figure $2 \mathrm{~h}$ ). The observed response of this architecture indicates that the photocurrent could not be solely attributed to direct regeneration of dye molecules by the evaporated contact since charge injection does not occur on $\mathrm{Al}_{2} \mathrm{O}_{3}$. Furthermore, the addition of the electron blocking layer resulted in values of $V_{o c}$ up to $0.86 \mathrm{~V}$ for forward scan $j-V$ (scanning from negative to positive voltage values) and $0.97 \mathrm{~V}$ for the reverse scan case. The increase in the $\mathrm{V}_{\text {oc }}$ on addition of the $\mathrm{Al}_{2} \mathrm{O}_{3}$ electron blocking layer can be attributed in part to the reduction of dark current arising because electrons can no longer undergo surface recombination with holes at the metal interface. Note that we also used an Au contact for this device which nominally has a higher work function than silver. However devices made without the $\mathrm{Al}_{2} \mathrm{O}_{3}$ blocking layer using $\mathrm{Au}$ as the contact showed lower $V_{o c}$ values than with $\mathrm{Ag}$.

The observed electrical bias dependence is unlikely to be related to variation in the rate of electron injection from the dye since transient absorption and photoluminescence studies have repeatedly demonstrated highly efficient injection from sensitized $\mathrm{TiO}_{2}$ films in air. ${ }^{[15],[16],[17]}$ Given the clear rectification observed in the dark current, the increase in photocurrent with reverse bias is instead likely to be related to E-field assisted photo charge collection within the devices. In this case there is significant competition between electron-oxidized dye recombination and the transport of the charge carriers by diffusion and drift in the device. This is in stark contrast to standard liquid or solid state DSSCs where the infiltrated hole transport medium can be considered as highly $p$-type. The high density of background holes and ionic additives in the pores screens charges within the $\mathrm{TiO}_{2}$ such that electron transport is dominated by diffusion. ${ }^{[18],[19],[20],[21],[22]}$ Consequently field-dependent charge collection is not normally seen in devices with electrolytes or organic (doped) hole transporting media. We also note that a device infiltrated with an inert electrolyte, containing no redox couple, showed reduced photovoltage and photocurrent relative to air (see supporting information). 
The comparatively high photocurrents observed at reverse bias relative to short circuit in dye sensitized dry cells (figure 2 ) indicate significant recombination occurs during charge collection at short circuit. To explore the nature of the charge carrier losses it is useful to examine the wavelength dependence of the photocurrent response as well as the transient optoelectronic properties of the devices. We applied a simple model to analyse the IPCE to assess the position and depths of the regions from which charges are collected in the devices at short circuit based on the optical penetration depth of different wavelengths (see supporting information). Some of the IPCE spectra in figure 2 show antibatic character in particular for FTO side illumination. The IPCE peaks corresponding to wavelengths where the dye absorptance is lower than $50 \%$ suggest remarkable internal quantum efficiency. The general shape of these IPCE spectra relative to the absorbtance indicate that the devices show a lower internal quantum efficiency at wavelengths that are absorbed most strongly (with a short penetration depth, $L_{a b s}$ ) than at weakly absorbed wavelengths (with a long penetration depth). Our analysis shows that charges generated in an intermediate region just below the metal contact are collected most efficiently. We estimate this region extends for several hundred $\mathrm{nm}$ within the film at short circuit (see supporting information) and is possibly due to the presence of a space charge layer where field assisted collection occurs. However, although charge collection from the region close to the metal contact is more efficient our simple model of the IPCE data also implies inefficient charge collection for the very top $200-300 \mathrm{~nm}$ of the film (e.g. see the antibatic behavior of the IPCE in figure $2 \mathrm{~d}$ for $\mathrm{Ag}$ side illumination, as well as model in supporting information). This could be explained considering enhanced excited state quenching for dyes in contact with Ag or surface recombination at the $\mathrm{Ag}$ interface. We note that some of this Ag is likely to be present as disconnected 'islands' which would extend into the top layer of the film and mediate recombination. Collection is also poor from the bottom region close to the FTO, consistent with the presence of a region where recombination is more likely in the absence of significant electric field.

Figure 4a shows photocurrent transients generated by a square wave of strongly absorbed $(420 \mathrm{~nm})$ and weakly absorbed $(550 \mathrm{~nm})$ monochromatic light superimposed on a white bias light. On illumination from the FTO side with strongly absorbed light the photocurrent initially steps up and then increases further towards a plateau with a time constant of $\tau_{S P C} \sim 0.1 \mathrm{~s}$, a decay with similar dynamics is observed when the light is turned off. These observations can be rationalized by considering the slow rise as being related to the transit time for the holes to diffuse to a region close to the Ag contact (as described above) where collection may be field assisted. The time scale is broadly consistent with observed diffusion coefficients for the holes. Considering $100 \mathrm{~nm}$ to $1 \mu \mathrm{m}$ as approximate range of the characteristic length in the system and diffusion time in the order of $0.1 \mathrm{~s}$ would correspond to apparent diffusion coefficients in the order of $10^{-9}$ to $10^{-7} \mathrm{~cm}^{2} \mathrm{~s}^{-1}$. This range of values is similar to those previously reported for hole hopping between dyes on $\mathrm{TiO}_{2}$ immersed in acetonitrile based electrolytes. ${ }^{[12]}$ Conversely, illuminating with the more uniformly absorbed light results in an immediate spike in the photocurrent which then decays. This could be attributed to collection of charge from the space charge region. The decay of the photocurrent following the initial step could be due to a gradual build-up of additional trapped charges which would increase the rate of recombination, reducing the collection efficiency. During the transients at both wavelengths the redistribution of trapped charge in the device is likely to change the photocurrent collection efficiency. The inset in figure 4a shows the time constant for a small perturbation transient photocurrent measurement under similar conditions using a weakly absorbed pulse. The time constant, $\tau_{T P C} \sim 10 \mu \mathrm{s}$, is consistent with a fast component to the photocurrent rise and fall in the stepped light measurements which we note is likely to be dominated by recombination and collection from a region close to the Ag (see IPCE analysis above). 

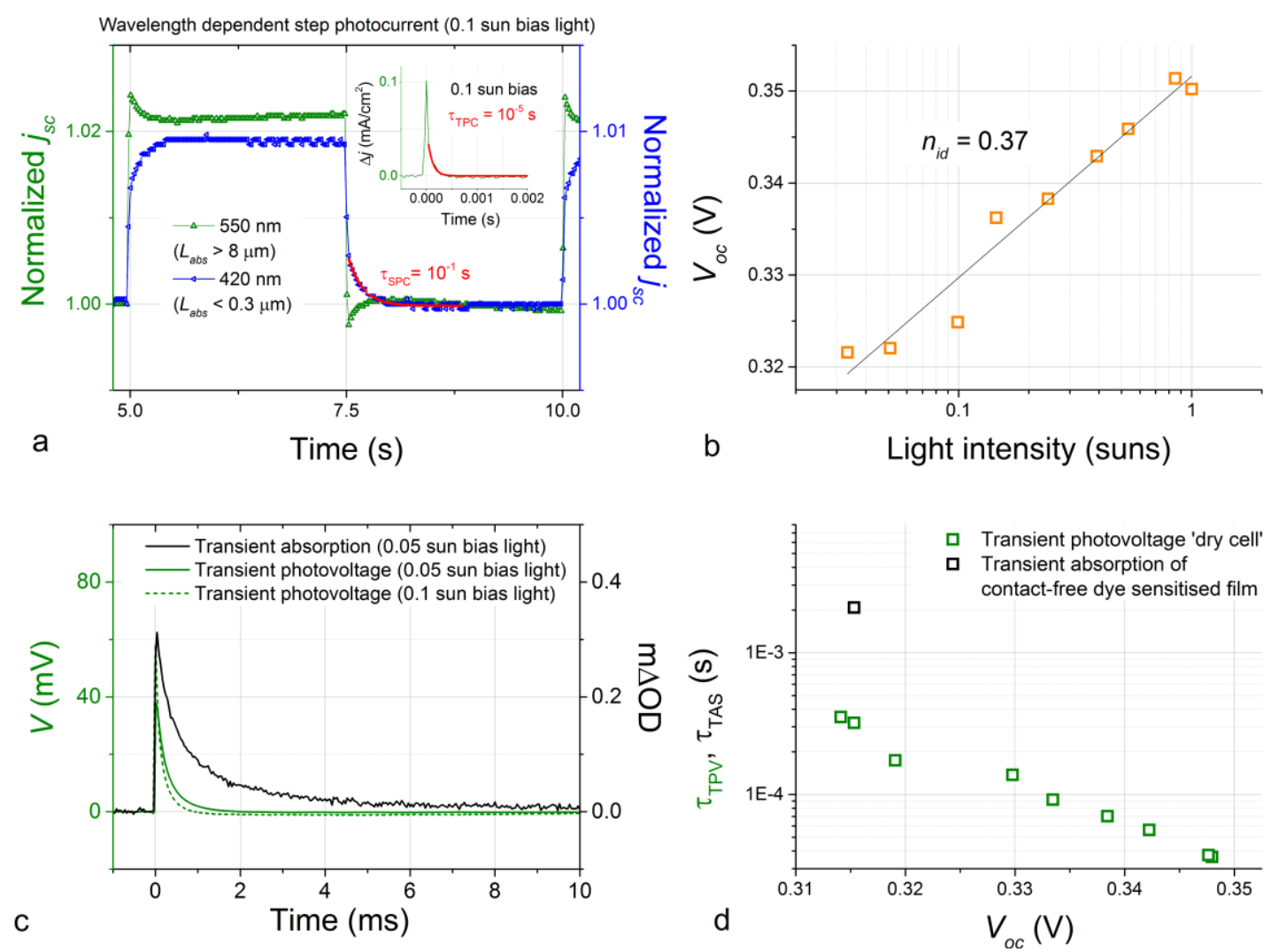

Figure 4. (a) Example of photocurrent transients under a square wave of monochromatic light applied to a $2 \mu \mathrm{m}$ thick D131 sensitized $\mathrm{TiO}_{2}$ dry cell. The strongly absorbed $420 \mathrm{~nm}$ and weakly absorbed 550 $\mathrm{nm}$ wavelengths (absorption length $L_{\text {abs }}$ is specified in the legend) were used as square wave small perturbation with a white LED bias light (intensity 0.1 sun equivalent). The inset shows a small perturbation photocurrent transient generated with a short green pulse (weakly absorbed) with a similar bias light intensity. (b) Example of $V_{o c}$ vs light intensity for a D131 dry cell. (c) Transient photovoltage following a short green excitation pulse and transient absorption decay monitoring the D131 cation absorption at $1000 \mathrm{~nm}^{[23]}$ upon laser excitation at $450 \mathrm{~nm}$ of a contact free dyed $\mathrm{TiO}_{2}$ film in air. Both measurements were performed using a white bias light. (d) Time constants obtained from transient photovoltage as a function of $V_{o c}$ and from transient absorption spectroscopy. The latter is plotted against the voltage referred to an equivalent bias light intensity.

To examine the nature of recombination at open circuit in our standard 'dry cell' architecture we assessed the light intensity dependence of the $V_{o c}$. The slope of this curve indicates an ideality factor of considerably less than 1 , suggesting that surface recombination may be the dominant route for carrier loss at open circuit (figure $4 \mathrm{~b}$ ). ${ }^{[24]}$ The relaxation time constants of small perturbation transient photovoltage decays at these intensities are shown figure 4c. These appear to be faster than those observed using transient absorption spectroscopy to monitor recombination of the photogenerated population of oxidized dyes (holes) on dyed- $\mathrm{TiO}_{2}$ films with no electrical contacts under similar illumination conditions (d). This further suggests that recombination at open circuit is strongly mediated by contacts, and implies that optimization of the interface materials in these devices could result in significant improvements in performance.

To summarize, we have demonstrated the direct collection of photogenerated holes by monolayers of dye molecules. The 'dry cell' concept presented here stands out from previously investigated DSSC architectures in that no dye regeneration step is needed in the device's active layer in order to implement hole collection. To our knowledge, this is the first report on self-regeneration by dye monolayers upon photo-oxidation. At present the performance of the devices appear limited by the competition between field assisted charge transport and electron-dye recombination. This demonstration illustrates the potential of molecular conductive coatings using multi-functional molecules, and suggests interesting new possibilities for the construction of organic electronic devices and sensors. 


\section{Experimental section}

Sample and device fabrication: 'dry' solar cells have been fabricated following the method described in Ref. ${ }^{[22]}$ FTO TEC15 substrates (15 $\Omega \mathrm{cm}^{-1}$ purchased from Pilkington) were patterned by etching with zinc powder and $2 \mathrm{M} \mathrm{HCl}$. The patterned substrates were washed with Hellmanex ( $2 \%$ in deionized water), deionized water, acetone and ethanol. This was followed by a 10 min oxygen plasma cleaning step to remove organic residues. A compact $\mathrm{TiO}_{2}$ layer (between 50 and $100 \mathrm{~nm}$ ) was deposited via spray pyrolysis at $300^{\circ} \mathrm{C}$ from a solution of titanium diisopropoxide bis(acetylacetonate) ( $75 \mathrm{wt} \%$ in isopropanol) diluted at $10 \mathrm{vol} \%$ in ethanol. The spray gun used was fed with compressed air. Mesoporous TiO2 films were deposited via screen printing. Dyesol 18NR-T paste was used. The thickness of the films was measured using a Dektak profilometer and was between 1.2 and $1.5 \mu \mathrm{m}$ for 'dry cells' and $0.9 \mu \mathrm{m}$ for "dry cells" including an alumina layer. The $\mathrm{TiO}_{2}$ films were heated to $450^{\circ} \mathrm{C}$ (temperature ramp over 2 hours) and sintered for 30 minutes in air. $\mathrm{TiCl}_{4}$ treatment was carried out by immersing the samples in $0.015 \mathrm{M} \mathrm{TiCl} 4$ aqueous solution for 45 minutes at $70^{\circ} \mathrm{C}$, and by repeating a sintering step at $450^{\circ} \mathrm{C}$ for 45 minutes. For devices including an $\mathrm{Al}_{2} \mathrm{O}_{3}$ buffer layer, this was obtained by spin coating a homemade paste of Alumina nanoparticles. A commercial (from Sigma Aldrich) aluminum oxide $(<50 \mathrm{~nm}$ ) dispersion $10 \mathrm{wt} \%$ in water was washed by centrifuging at 7500 RPM for 6 hours and redispersing in absolute ethanol with an ultrasonic probe at a duty cycle of $50 \%$ for a duration of 5 minutes. This was repeated three times. A paste was then made with the following composition $1 \mathrm{~g} \mathrm{Al}_{2} \mathrm{O}_{3}$ to $3.33 \mathrm{~g}$ of $\alpha$-terpineol to $5 \mathrm{~g}$ of a $50: 50$ mix of ethyl-cellulose $10 \mathrm{cP}$ and $46 \mathrm{cP}$ in ethanol $10 \mathrm{wt} \%$. After each addition, the mix was stirred for 2 minutes and sonicated for one minute. Finally, the resulting mixture was introduced into a rotary evaporator to remove excess ethanol in order achieve the required paste consistency suitable for doctor blading, spincoating or screen-printing. Sintering at $450^{\circ} \mathrm{C}$ for 45 minutes of the film resulted in a $150 \mathrm{~nm}$ thick layer of mesoporous $\mathrm{Al}_{2} \mathrm{O}_{3}$. The substrates were then cooled down to $70^{\circ} \mathrm{C}$ before immersion in dye solutions for 3 hours. $0.1 \mathrm{mM}$ D131 and $0.06 \mathrm{mM}$ of D149 dyes were dissolved in a 1:1 mixture of acetonitrile and tertbutyl alcohol and used to dye the $\mathrm{TiO}_{2}$ and the $\mathrm{TiO}_{2} / \mathrm{Al}_{2} \mathrm{O}_{3}$ structures. $40 \mathrm{~nm}$ of silver or gold were evaporated on top of the dyed mesostructures to attain semitransparent contacts to enable the measurement of external quantum efficiency when illuminating the device through the metal electrode.

Electrical and photovoltaic characterisation of solar cells: current voltage measurements under solarsimulated AM 1.5 sunlight were performed using an ABET solar simulator calibrated to give $100 \mathrm{~mW} \mathrm{~cm}^{-2}$, an NREL-calibrated KG5 filtered silicon reference cell and a sourcemeter (Keithley 2400, USA) for the electrical measurement. I-V curves were measured at a scan rate of $0.15 \mathrm{~V} \mathrm{~s}^{-1}$ starting with a forward scan (increasing voltage) followed by a reverse scan. IPCE was measured by illuminating the samples with a chopped monochromatic light (frequency $3 \mathrm{~Hz}$ ). A background bias light was applied using light emitting diodes at an equivalent solar irradiance of $10 \%$ sun. The measurement was performed by exposing either the FTO glass side or the metal side of the solar cells to the light sources. Devices were masked with aperture of $0.09 \mathrm{~cm}^{2}$ for all optoelectronic measurements. All measurements of $j-V$ and IPCE were carried out in air unless stated otherwise. When a solvent or electrolyte was introduced in the device (see supporting information), this was dispensed directly on the cell. The substrate was then covered with a glass cover slip before the start of the measurement. Optoelectronic transient and transient absorption measurements were carried out as described previously, using either a green LED or laser pulse to generate the perturbations on top of a white bias light generated by LEDs. ${ }^{[21],[25]}$

\section{Acknowledgements}

We thank Brian O'Regan and Thomas Kirchartz for helpful discussion. DM and PB are grateful for EPSRC fellowship EP/J002305/1. 


\section{References}

[1] T. A. Heimer, S. T. D. Arcangelis, F. Farzad, J. M. Stipkala, G. J. Meyer, Inorg. Chem. 1996, 1669, 5319.

[2] P. Bonhôte, E. Gogniat, S. Tingry, C. Barbe, N. Vlachopoulos, F. Lenzmann, P. Comte, M. Grätzel, J. Phys. Chem. B 1998, 5647, 1498.

[3] S. Ardo, G. J. Meyer, J. Am. Chem. Soc. 2010, 132, 9283.

[4] Q. Wang, N. Evans, S. M. Zakeeruddin, I. Exnar, M. Grätzel, J. Am. Chem. Soc. 2007, 129, 3163.

[5] S. Ardo, G. J. Meyer, J. Am. Chem. Soc. 2011, 133, 15384.

[6] U. Bach, Y. Tachibana, J. Moser, S. A. Haque, J. R. Durrant, M. Grätzel, J. Am. Chem. Soc. 1999, 2, 7445.

[7] Q. Wang, S. M. Zakeeruddin, M. K. Nazeeruddin, R. Humphry-Baker, M. Grätzel, J. Am. Chem. Soc. 2006, 128, 4446.

[8] J. Melas-Kyriazi, I.-K. Ding, A. Marchioro, A. Punzi, B. E. Hardin, G. F. Burkhard, N. Tétreault, M. Grätzel, J.-E. Moser, M. D. McGehee, Adv. Energy Mater. 2011, 1, 407.

[9] L. Yang, U. B. Cappel, E. L. Unger, M. Karlsson, K. M. Karlsson, E. Gabrielsson, L. Sun, G. Boschloo, A. Hagfeldt, E. M. J. Johansson, Phys. Chem. Chem. Phys. 2012, 14, 779.

[10] C. T. Weisspfennig, D. J. Hollman, C. Menelaou, S. D. Stranks, H. J. Joyce, M. B. Johnston, H. J. Snaith, L. M. Herz, Adv. Funct. Mater. 2013, 24, 668.

[11] G. Boschloo, T. Marinado, K. Nonomura, T. Edvinsson, A. G. Agrios, D. P. Hagberg, L. Sun, M. Quintana, C. S. Karthikeyan, M. Thelakkat, A. Hagfeldt, Thin Solid Films 2008, 516, 7214.

[12] D. Moia, V. Vaissier, I. López-Duarte, T. Torres, M. K. Nazeeruddin, B. C. O’Regan, J. Nelson, P. R. F. Barnes, Chem. Sci. 2014, 5, 281.

[13] V. Vaissier, E. Mosconi, D. Moia, M. Pastore, J. M. Frost, F. De Angelis, P. R. F. Barnes, J. Nelson, Chem. Mater. 2014, 4731.

[14] F. O. Lenzmann, B. C. O’Regan, J. J. T. Smits, H. P. C. E. Kuipers, P. M. Sommeling, L. H. Slooff, J. a. M. van Roosmalen, Prog. Photovoltaics Res. Appl. 2005, 13, 333.

[15] J. E. Kroeze, N. Hirata, L. Schmidt-Mende, C. Orizu, S. D. Ogier, K. Carr, M. Grätzel, J. R. Durrant, Adv. Funct. Mater. 2006, 16, 1832.

[16] R. J. Ellingson, J. B. Asbury, S. Ferrere, H. N. Ghosh, J. R. Sprague, T. Lian, A. J. Nozik, J. Phys. Chem. B 1998, 2, 6455 .

[17] S. K. Pathak, A. Abate, T. Leijtens, D. J. Hollman, J. Teuscher, L. Pazos, P. Docampo, U. Steiner, H. J. Snaith, Adv. Energy Mater. 2014, n/a.

[18] B. O’Regan, J. Moser, M. Anderson, M. Grätzel, J. Phys. Chem. 1990, 94, 8720.

[19] F. Fabregat-Santiago, J. Bisquert, L. Cevey, P. Chen, M. Wang, S. M. Zakeeruddin, M. Grätzel, J. Am. Chem. Soc. 2009, 131, 558. 
[20] P. R. F. Barnes, A. Y. Anderson, S. E. Koops, J. R. Durrant, B. C. O’Regan, J. Phys. Chem. C 2009, 113, 1126.

[21] P. R. F. Barnes, K. Miettunen, X. Li, A. Y. Anderson, T. Bessho, M. Grätzel, B. C. O’Regan, Adv. Mater. 2013, 25, 1881.

[22] A. Abate, T. Leijtens, S. Pathak, J. Teuscher, R. Avolio, M. E. Errico, J. Kirkpatrik, J. M. Ball, P. Docampo, I. McPherson, H. J. Snaith, Phys. Chem. Chem. Phys. 2013, 15, 2572.

[23] A. Fattori, L. M. Peter, H. Wang, H. Miura, F. Marken, J. Phys. Chem. C 2010, 205, 11822.

[24] T. Kirchartz, F. Deledalle, P. S. Tuladhar, J. R. Durrant, J. Nelson, J. Phys. Chem. Lett. 2013, 4, 2371.

[25] A. Y. Anderson, P. R. F. Barnes, J. R. Durrant, B. C. O’Regan, J. Phys. Chem. C 2010, 1953. 